\title{
Prediction of drought related transcripts in cotton (Gossypium hirsutum): an in- silico approach
}

\author{
Noor-us-Sabah ${ }^{1}$, Mahmood-ur-Rahman ${ }^{1 *}$, Tayyaba Shaheen ${ }^{1}$, Shazia \\ Anwer Bukhari ${ }^{2}$, Muhammad Qasim¹, Muhammad Shareef Masoud ${ }^{1}$ and \\ Khadim Hussain ${ }^{1}$
}

${ }^{1}$ Department of Bioinformatics and Biotechnology, GC University - Faisalabad, Pakistan
${ }^{2}$ Department of Applied Chemistry and Biochemistry, GC University - Faisalabad, Pakistan
*Corresponding author email: mahmoodansari@gcuf.edu.pk

\section{Citation}

Noor-us-Sabah, Mahmood-ur-Rahman, Tayyaba Shaheen, Shazia Anwer Bukhari, Muhammad Qasim, Muhammad Shareef Masoud and Khadim Hussain. Prediction of drought related transcripts in cotton (Gossypium hirsutum): an in-silico approach. Pure and Applied Biology. Vol. 4, Issue 2, 2015, pp 244-251

Received: $29 / 03 / 2015$

Revised: 03/05/2015

Accepted: 10/05/2015

\section{Abstract}

Prediction of candidate transcripts using bioinformatics tools is an exciting domain which saves the time of a researcher. Drought responsive genes were identified using homology method. The known genes from sequenced organisms (like Arabidopsis, rice, etc.) were compared with ESTs of different crops from various databases. Total 177 genes of Arabidopsis and rice with known functions were retrieved from different databases. The genes were classified and their role in drought was characterized on the basis of already available literature. After classification, 110 genes were selected for further studies. mRNA sequences and accession numbers of the 110 drought tolerant genes were retrieved which were analyzed using BLAST tool against ESTs on "NCBI" databases. ESTs in different crops (like cotton, wheat, potato, maize, sorghum, etc.) were found and data regarding ESTs from cotton were retrieved. Total 9096 ESTs were analyzed from NCBI. The 26 short listed genes were analyzed for "Multiple Sequence Alignment" and aligned with ESTs which were identified earlier. They showed considerable homology with ESTs. All the genes were thoroughly searched for their novelty and finally 8 genes were reported as candidate genes responsible for drought stress tolerance.

Keyword: Bioinformatics; Cotton; Drought; ESTs; Transcripts

\section{Introduction}

Drought stress usually happens in atmospheric conditions where the water is continuously lost by the process of evaporation and transpiration and as a result the water level in the soil is decreased. Drought is one of the most prominent natural environmental hazard which affects 
large number of people directly [1, 2]. Almost all climatic regions are affected by drought [2] and every year almost half of the regions of the world are vulnerable to the threat of drought [3]. In many countries, drought stress is also an obstinate environmental obstacle. In the last few years, the disaster incidences from drought stress are pointedly increased without any reported evidences of increased drought severity [2].

Drought tolerance is observed in almost all plant species but the extent of response is variable from specie to specie and sometimes even with in the species [4]. Drought affects plants in different ways. Severe drought may result in the disruption of metabolism, seizure of photosynthesis and finally it can lead to the plants death [4]. It has been observed that drought stress plays a vital role as a limiting factor on some initial phases of plant growth and establishment [5]. Water stress hinders enlargement of cell, elongation of cell and cell division. It can reduce the growth of plant by the disturbance in many biochemical and physiological processes [6]. Identification of genes responsible for drought is an important strategy to combat the serious problem. Once the genes are identified, they can be used to develop genetically engineered plants having resistance to drought. Using bioinformatics tools, the transcripts/genes responsible for drought stress can be identified. In this study, the drought related transcripts in cotton were identified using bioinformatics tools. The procedure is less laborious, less time consuming and it requires less resources. Study of genes related to drought stress in cotton and there identification is of key importance to understand the mechanism of drought tolerance and can help in increasing the cotton production which will ultimately contribute towards the national economy.

\section{Materials and Methods}

Retrieval of Drought Tolerant genes of Different Plant Species

The previously reported drought tolerant genes from different plants i.e., Arabidopsis thaliana, rice wheat and soya been were searched from various scholarly articles and then their accession numbers and sequences in FASTA format were retrieved from different data bases like NCBI (www.ncbi.nlm.nih.gov/), $\quad$ TAIR (www.arabidopsis.org/), GRAMENE (www.gramene.org/), TIGR (www.jcvi.org), etc.

\section{Nucleotide BLAST of drought tolerant genes}

The retrieved genes were subject to BLAST (http://blast.ncbi.nlm.nih.gov/Blast.cgi) and the identified ESTs were saved for further analysis.

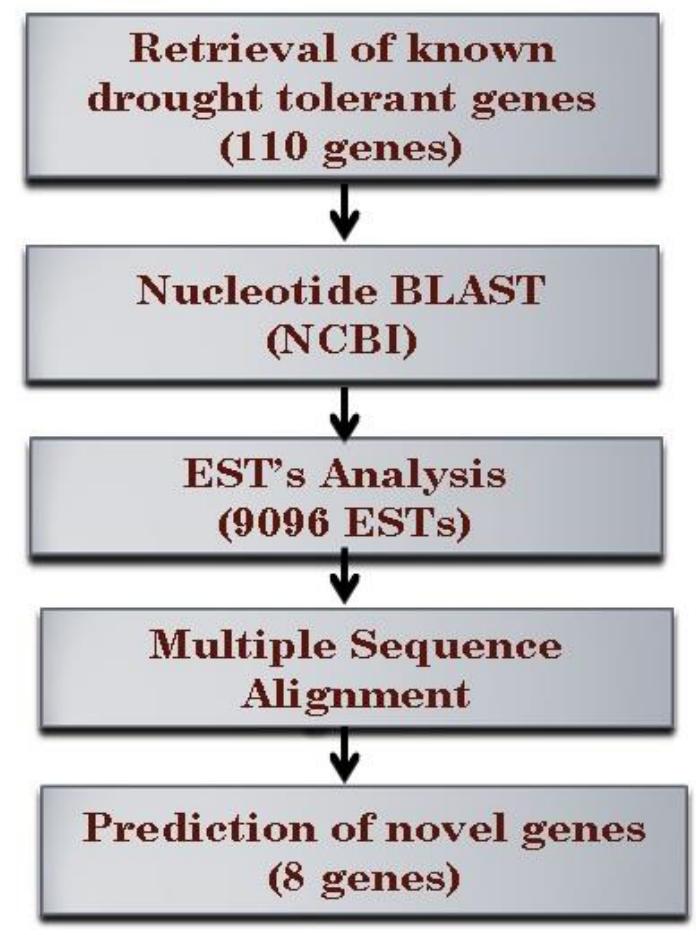

Figure-1. Flow chart summary of methods used in prediction of drought related transcripts in cotton 
Multiple Sequence Alignment of ESTs Multiple Sequence Alignment was carried out at EMBL-EBI web portal (http://www.ebi.ac.uk/Tools/msa/clustalw2/) and CUSTAL OMEGA software was used which is freely available online. The potential candidate genes/transcripts were searched in the literature for their possible homologues in cotton. Only the novel genes/transcripts not identified previously were reported in this article. The detailed scheme of the methodology is given in Figure-1.

\section{Results}

\section{Retrieval of Genes}

The known drought tolerant genes were retrieved from various plant species including Arabidopsis thaliana, Hordeum vulgare, Glycine max, Solanum lycopersicum, Oryza sativa, Triticum aestivum, Zea mays, etc. Total 177 genes were retrieved, cataloged and their functions were listed. On the basis of their functions and involvement in drought stress tolerance mechanism, 110 genes were short listed and included in present study (Table-1). Sequence Alignment and ESTs analysis After nucleotide and protein BLAST of 110 genes, the 9096 ESTs were identified in cotton (Gossypium hirsutum). The number of ESTs against various plant species identified in cotton are being shown in Figure-2. Maximum 429 cotton ESTs were identified in genes of Glycine max origin followed by 273 in Triticum aestivum, 181 in Solanum lycopersicum and 179 in Arabidopsis thaliana. ESTs in other plant species were also found in cotton.

Multiple Sequence Alignment of cotton ESTs

Multiple sequence alignment of the EST's from cotton was carried out with the respective genes and sequence similarities were found. Total of 26 genes were aligned and their sequence similarities were found.
On the basis of significant similarities, 19 genes were short listed as potential novel genes in cotton. Genes which were aligned with cotton were then searched in literature and 8 genes were found not to be known previously in cotton (Table-2).

\section{Discussion}

Identification of genes/transcripts using bioinformatics tools is very popular now days. Because of the availability of large amount of information and complete or partial genome sequence of Arabidopsis thaliana, Hordeum vulgare, Glycine max, Solanum lycopersicum, Triticum aestivum, Zea mays and Oryza sativa, which could be used to identify novel genes. New gene discovery approach requires searching of whole genome sequence for homologous sequences of known genes or known gene families by in silico methods. Recent researches prove the proficiency of this in silco technique of finding new genes $[7,8]$. All the nucleotide and protein sequences of 143 were aligned using BLASTn and BLASTp respectively. Out of these 110 genes 26 genes aligned with cotton genome which proved the presence of drought tolerant genes in cotton.

In this study, we report 8 novel genes which are supposed to play important role in drought stress tolerance in cotton. These genes are known for the said function in other plants and are well characterized. ABF3 is identified in Arabidopsis which is responsible for transcription activation [9]. It is also know as a DNA binding protein gene and is a transcription factor. STRS1 is also identified in Arabidopsis thaliana and is known as a stress response suppressor [10]. It is involved in DNA replication. SEP2 is a stress enhanced protein and is identified in Arabidopsis thaliana [11]. WAPX1 is reported in Triticum aestivum and codes for ascorbate peroxidase enzyme which catalyse the conversion of $\mathrm{H}_{2} \mathrm{O}_{2}$ to $\mathrm{H}_{2} \mathrm{O}$ [12]. 
Sabah et al.
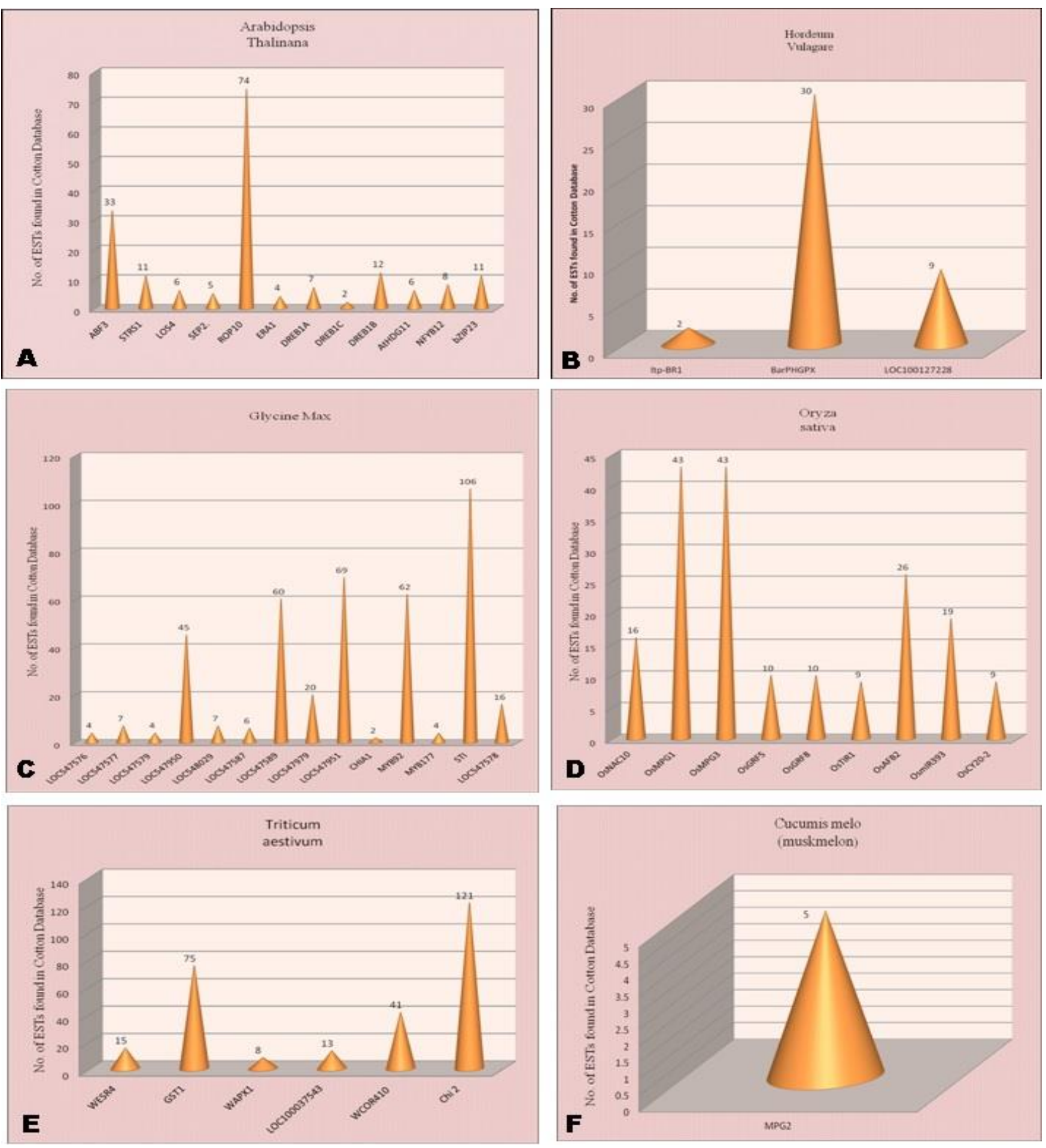

Figure-2. Cotton ESTs identified against genes already known in different plant species 
Table-1: Genes responsible for drought tolerance in different Plants species

\begin{tabular}{|c|c|c|c|c|c|c|c|}
\hline S. No. & Gene Name & Accession No. & Source & S. No. & Gene Name & Accession No. & Source \\
\hline 1. & HSA32 & NM_118250.4 & Arabidopsis thaliana & 2. & HRD & DQ446604.1 & Arabidopsis Thaliana \\
\hline 3. & DREB2A & NM_001036760.1 & Arabidopsis thaliana & 4. & rice adc & AY604047.1 & Oryza sativa \\
\hline 5. & ABF3 & NM_001036708.3 & Arabidopsis thaliana & 6. & SB & JN661889.1 & Oryza sativa \\
\hline 7. & STRS1 & NM_102933.2 & Arabidopsis thaliana & 8. & OsMPG1 & JN408497.1 & Oryza sativa \\
\hline 9. & ERD15 & NM_201929.2 & Arabidopsis thaliana & 10. & MPG2 & AF062466.1 & Cucumis melo \\
\hline 11. & LOS4 & NM_115171.3 & Arabidopsis thaliana & 12. & OsMPG3 & JN408499.1 & Oryza sativa \\
\hline 13. & SEP2 & NM_127766.2 & Arabidopsis thaliana & 14. & OsGRF5 & BK004860.1 & Oryza sativa \\
\hline 17. & ROP10 & NM_114673.2 & Arabidopsis thaliana & 18. & OsGRF8 & BK004863.1 & Oryza sativa \\
\hline 19. & HOS10 & NM_179418.1 & Arabidopsis thaliana & 20. & NFYB12 & AK227164.1 & Arabidopsis Thaliana \\
\hline 21. & ERD15 & NM_180019.2 & Arabidopsis thaliana & 22. & DST & GQ178286.1 & Oryza sativa \\
\hline 23. & ERD15 & NM_201928.2 & Arabidopsis thaliana & 24. & AtHDG11 & NM_105996.3 & Arabidopsis thaliana \\
\hline 25. & ERD15 & NM_201929.2 & Arabidopsis thaliana & 26. & OsTIR1 & EU400583.1 & Oryza sativa \\
\hline 27. & DREB2B & NM_001036760.1 & Arachis hypogaea & 28. & OsAFB2 & NM_113593.2 & Arabidopsis thaliana \\
\hline 29. & DREB2A & AB007790.1 & Arabidopsis thaliana & 30. & OsmIR393 & NM_101152.4 & Arabidopsis thaliana \\
\hline 31. & ltp-BR1 & U88090.1 & Hordeum vulgare & 32. & WR1 & M94959.1 & Triticum aestivum \\
\hline 35. & HSP90 & AY325266.1 & Hordeum vulgare & 36. & bZIP23 & BT029022.1 & Arabidopsis thaliana \\
\hline 37. & PHGPX & AB096704.1 & Hordeum vulgare & 38. & RD22-like & NM_122472.3 & Glycine max \\
\hline 39. & CHIA1 & AF202731.1 & Glycine max & 40. & RD29B-like & NM_001198228.1 & Glycine max \\
\hline 41. & MYB92 & DQ822903.1 & Glycine max & 42. & ARR5 & NP_190393.1 & Arabidopsis thaliana \\
\hline 43. & MYB177 & DQ822925.1 & Glycine max & 44. & ARR7 & NM_101763.2 & Arabidopsis thaliana \\
\hline 45. & STI & $\mathrm{X} 79770.1$ & Glycine max & 46. & ARR15 & NM_106147.1 & Arabidopsis thaliana \\
\hline 47. & DD4/62 & AJ289155.1 & Glycine max & 48. & ARR22 & NM_180180.2 & Arabidopsis thaliana \\
\hline 49. & DREB3 & AF506825.1 & Solanum lycopersicum & 50. & NHX1 & AF056190.1 & Arabidopsis thaliana \\
\hline 51. & rps4 & YP_514854.1 & Solanum lycopersicum & 52. & SOS1 & NM_126259.3 & Arabidopsis thaliana \\
\hline 53. & RbcL & YP_514860.1 & Solanum lycopersicum & 54. & P5CS1 & NM_001202786.1 & Arabidopsis thaliana \\
\hline 55. & WESR4 & $\mathrm{AB} 011445.1$ & Triticum aestivum & 56. & RD29A & NM_124610.2 & Arabidopsis thaliana \\
\hline 57. & Cht2 & AF112963.1 & Triticum aestivum & 58. & AtPCS1 & NM_120297.3 & Arabidopsis thaliana \\
\hline 59. & Cht4 & AF112963.1 & Triticum aestivum & 60. & ZmSNAC1 & EU963406.1 & Zea Mays \\
\hline 61. & GST1 & AF184059.1 & Triticum aestivum & 62. & ABCG22 & XM_641139.1 & Dictyostelium discoideum \\
\hline 63. & GST1 & AF387085.1 & Triticum aestivum & 64. & PYL5 & NM_120626.2 & Arabidopsis thaliana \\
\hline 65. & Tatil & AY077702.1 & Triticum aestivum & 66. & KfVP1 & FJ176929.1 & GRAPE \\
\hline 73. & Chi 2 & AB029935.1 & Triticum aestivum & 74. & SnRK2 & NM_001203118.2 & Arabidopsis Thaliana \\
\hline
\end{tabular}




\begin{tabular}{|c|c|c|c|c|c|c|c|}
\hline 75. & Chi 3 & AB029936.1 & Triticum aestivum & 76. & AtNCED3 & NM_112304.2 & Arabidopsis Thaliana \\
\hline 77. & hsp18a & EU952864.1 & Zea mays & 78. & LTI65 & NM_124609.3 & Arabidopsis Thaliana \\
\hline 79. & gst1 & M16901.1 & Zea mays & 80. & RNS1 & NM_126351.2 & Arabidopsis Thaliana \\
\hline 81. & gpa1 & X07157.1 & Zea mays & 82. & AtDiB & NM_130216.2 & Arabidopsis Thaliana \\
\hline 85. & glu1 & U25157.1 & Zea mays & 86. & AtD121 & NM_117683.4 & Arabidopsis Thaliana \\
\hline 87. & hsp22 & EU961071.1 & Zea mays & 88. & AtDr4 & NM_105993.2 & Arabidopsis Thaliana \\
\hline 89. & $\mathrm{dbf} 3$ & AF450481.1 & Zea mays & 90. & CBF4 & NM_124578.1 & Arabidopsis Thaliana \\
\hline 91. & hsp18f & X54076.1 & Zea mays & 92. & Atlea3 & NM_001203153.1 & Arabidopsis Thaliana \\
\hline 93. & hsp26 & EU964533.1 & Zea mays & 94. & AtP5CS2 & NM_115419.4 & Arabidopsis Thaliana \\
\hline 95. & OsNAC10 & JQ974964.1 & Oryza sativa & 96. & AtProT1 & NM_001084556.1 & Arabidopsis Thaliana \\
\hline 97. & ERA1 & AF214106.1 & Arabidopsis Thaliana & 98. & AtAPX1 & AY094002.1 & Arabidopsis Thaliana \\
\hline 99. & LEA3 & DQ056621.1 & Arabidopsis Thaliana & 100. & AtSOS1 & NM_126259.3 & Arabidopsis Thaliana \\
\hline 101. & DREB1A & EF523125.1 & Arabidopsis Thaliana & 102. & AtNHX1 & NM_122597.2 & Arabidopsis Thaliana \\
\hline 103. & OsDREB2A & AB007790.1 & Arabidopsis Thaliana & 104. & Hua1 & NM_112103.3 & Arabidopsis thaliana \\
\hline 105. & DREB1C & EF523053.1 & Arabidopsis Thaliana & 106. & TaWRKY2 & EU665430.1 & Triticum aestivum \\
\hline 107. & DREB1B & AB007788 & Arabidopsis Thaliana & 108. & TaWRKY19 & EU665428.1 & Triticum aestivum \\
\hline
\end{tabular}

Table-2: Novel candidate genes predicted for drought tolerance in cotton (Gossypium hirsutum L.)

\begin{tabular}{|c|c|c|c|c|}
\hline S. No. & Gene Name & Accession No. & Origin & Gene Function \\
\hline 1. & AFB3 & NM_001036708.3 & Arabidopsis thaliana & $\begin{array}{l}\text { Abscisic acid responsive elements-binding factor-3, DNA binding/ protein, } \\
\text { transcription factor, transcription activator }\end{array}$ \\
\hline 2. & STRS1 & NM_102933.2 & Arabidopsis thaliana & Stress response suppressor-1, ATP-dependent helicase \\
\hline 3. & SEP2 & NM_127766.2 & Arabidopsis thaliana & Stress enhanced protein \\
\hline 4. & ltp-BR1 & U88090.1 & Hordeum vulgare & Nonspecific lipid transfer protein \\
\hline 5. & WAPX1 & AF387739.1 & Triticum aestivum & Ascorbate peroxidase \\
\hline 6. & ERA1 & AF214106.1 & Arabidopsis thaliana & Protein farnesyltransferase subunit beta \\
\hline 7. & AtProT1 & NM_001084556.1 & Arabidopsis thaliana & Proline transporter \\
\hline 8. & TaWRKY2 & EU665430.1 & Triticum aestivum & WRKY19 transcription factor \\
\hline
\end{tabular}


It is regulated in response to biotic and abiotic stress and is responsible to protect plant cell against adverse environmental conditions. ltp-BR1, ERA1, AtProT1 and TaWRKY2 were also predicted in this study which play role in drought stress tolerance in various plants [13-16].

Due to availability of large amount of sequence data in the various databases, it is very easy to predict the functions of some particular fragment of nucleotides. Genome sequencing made it even more reliable. It is easy, time saving, reliable and also saves resources. Number of genes has been identified by using in silico approaches. Here, we also report the identification of 8 drought tolerant genes in cotton by gene homology approach using various bioinformatics tools.

\section{Acknowledgements}

Financial support in the form of Start-up grant from Higher Education Commission, Islamabad is gratefully acknowledged.

\section{References}

1. Hewitt K (1997). Regions at Risk: A Geographical Introduction to Disasters. Addison-Wesley Longman, UK.

2. Wilhite DA \& Knutson CL (2008). Drought management planning: Conditions for success. Options Méditerranéennes 80(A): 141- 148.

3. Kogan FN (1997). Global drought watch from space. Bull Amer Meteor Soc 78(4): 621-636.

4. Jaleel CA, Manivannan $\mathrm{P}$, Wahid A, Farooq M, Al-Juburi HJ, Somasundaram R \& Vam RP (2009). Drought Stress in Plants: A Review on Morphological Characteristics and Pigments Composition. Int J Agric Biol 11(1): 100-105.

5. Shao HB, Chu LY, Jaleel CA \& Zhao CX (2008). Water-deficit stress-induced anatomical changes in higher plants. Cell Biol 331(3): 215-225.
6. Farooq M, Wahid A, Kobayashi N, Fujita D \& Basra SMA (2009). Plant drought stress: effects, mechanisms and management. Agron Sustain Dev 29: 185212.

7. Wittenberger T \& Schaller HC (2001). An expressed sequence tag (EST) data mining strategy succeeding in the discovery of new G-protein coupled receptors. Mol Biol 7(3): 799-813.

8. Bathgate RA, Samuel CS, Burazin TC, Layfield S, Claasz AA, Reytomas IG, Dawson NF, Zhao C, Bond C, Summers RJ, Parry LJ, Wade JD \& Tregear GW (2002). Human relaxin gene $3(\mathrm{H} 3)$ and the equivalent mouse relaxin (M3) gene. Novel members of the relaxin peptide family. $J$ Biol Chem 277(2): 1148-1157.

9. Yoshida T, Fujita Y, Sayama H, Kidokoro S, Maruyama K, Mizoi J, Shinozaki K \& Yamaguchi-Shinozaki K (2010). AREB1, AREB2, and ABF3 are master transcription factors that cooperatively regulate ABREdependent ABA signaling involved in drought stress tolerance and require $\mathrm{ABA}$ for full activation. Plant J 61(4): 672-85.

10. Khan A, Garbelli A, Grossi S, Florentin A, Batelli G, Acuna T, Zolla G, Kaye Y, Paul LK, Zhu JK, Maga G, Grafi G \& Barak $S$ (2014). The Arabidopsis Stress response suppressor DEAD-box RNA helicases are nucleolar- and chromocenter-localized proteins that undergo stress-mediated relocalization and are involved in epigenetic gene silencing. Plant J 79(1): 28-43.

11. Andersson U,Heddad M \& Adamska I (2003). Light Stress-Induced One-Helix Protein of the Chlorophyll $a / b$-Binding Family Associated with Photosystem. Plant Physiol 132(2): 811-820.

12. Caverzan A, Passaia G, Rosa SB, Ribeiro CW, Lazzarotto F \& MargisPinheiro M (2012). Plant responses to stresses: Role of ascorbate peroxidase in the 
antioxidant protection. Genet $\mathrm{Mol}$ Biol 35(4): 1011-1019.

13. Smith LJ, Roby Y, Allison JR \& van Gunsteren WF (2013). Molecular dynamics simulations of barley and maize lipid transfer proteins show different ligand binding preferences in agreement with experimental data. Biochem. 52(30): 502938.

14. Wang Y, Ying J, Kuzma M, Chalifoux M, Sample A, McArthur C, Uchacz T, Sarvas C, Wan J, Dennis DT, McCourt P \& Huang Y (2005). Molecular tailoring of farnesylation for plant drought tolerance and yield protection. Plant J 43(3): 413-24.
15. Grallath S, Weimar T, Meyer A, Gumy C, Suter-Grotemeyer M, Neuhaus JM \& Rentsch D (2005). The AtProT Family. Compatible Solute Transporters with Similar Substrate Specificity But Differential Expression Patterns. Plant Physiol 137(1): 117-126.

16. Niu CF, Wei W, Zhou QY, Tian AG, Hao YJ, Zhang WK, Ma B, Lin Q, Zhang ZB, Zhang JS \& Chen SY (2012). Wheat WRKY genes TaWRKY2 and TaWRKY19 regulate abiotic stress tolerance in transgenic Arabidopsis plants. Plant, Cell Environ 35(6):
1156-70. 